\title{
First Steps towards Localized Opening of the Blood-Brain-Barrier by IR Laser Illumination Through the Rodent Skull
}

https://doi.org/10.1515/cdbme-2019-0054

\begin{abstract}
Glioblastoma, an aggressive malign tumor of the brain, is one of the most shattering diagnoses due to its very poor prognosis and limited treatment options. These options mainly consist of surgical or radiation therapeutic removal of as much tumor mass as possible, which unfortunately is almost always incomplete. Even worse, chemotherapy is of little use, as the special setup of the brain's vessels severely limits the transit into the parenchyma of elsewhere efficient cytostatica. This Blood-Brain-Barrier (BBB) is for quite some time the target of sophisticated and nano-particle based transport mechanisms, however it is reported, that a boost of permeability for most of the brain can be achieved based on moderate temperature increase. One means to locally and reversibly increase the brain's temperature and thus potentially opening the BBB may be achieved by illuminating the skull with infrared laser light, thus causing punctual heating and heat diffusion into the cortex. In extension of the common laser light guiding by glass fibres, we use a micro-positioned simple optics to focus a 1470 $\mathrm{nm}$ laser beam of approximately $500 \mu \mathrm{m}$ in diameter on the skull. The apparent opening of the BBB is evidenced by the localized spread of Evans Blue injected into the tail vein of said rat, binding to Albumin $(64,6 \mathrm{kDa})$ in the body. This marker molecule is usually blocked from passing through the intact $\mathrm{BBB}$, but under IR illumination for half a minute, it appeared in post mortem visible blobs. Temperature profiles and potential tissue damage are now under investigation by high speed thermal camera and post mortem histology.
\end{abstract}

Keywords: Blood Brain Barrier Opening, Infrared Laser Illumination, Evans Blue.

\section{INTRODUCTION}

Nearly every effort of treating diseases affecting the brain with medication faces the challenges posed by the highly specialized makeup of intracerebral blood vessels. In contrast to the rest of the body, capillaries in the brain are lined with an array of different cell types that are meant to selectively

*Corresponding author: Paul Schlett, Section for Neuroelectronic Systems, Uniklinik Freiburg, Hugstetter Straße 55, Freiburg, Germany, e-mail: paul.schlett@uniklinik-freiburg.de

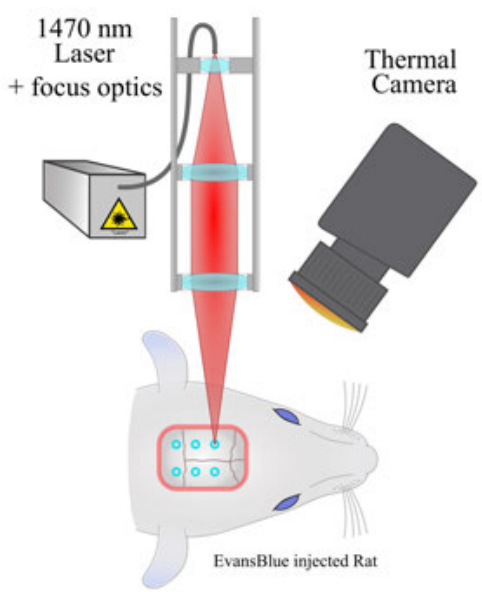

Fig. 1: Experimental setup: An infrared diode laser is coupled via a glass fiber to an optical lens assembly on a 3-axis micromanipulator providing a $538 \mu \mathrm{m}$ focus spot on the skull of an Evans Blue injected rat. Skull temperature is accurately monitored by a thermal camera.

exclude substances to traverse from the blood stream to the brain parenchyma. Merely small lipid soluble molecules can pass the Blood-Brain-Barrier (BBB) easily by passive diffusion and there are specific transport mechanisms for essential compounds such as glucose [1] . The integrity and selectivity of the BBB constitutes an essential physiological protection by keeping potentially harmful material from entering the brain. In that context effective transport of medication to a specific target in the central nervous system (CNS) is also blocked making it difficult to medicate various corresponding diseases. Among others, treatment of primary brain tumors with cytostatica is severely limited by the accessibility of the drug within the CNS which is unproblematic in the rest of the body. Even though glioblastoma cores are thought to not have a BBB, some part of the aggressively growing rim does [2]. The consequence is a devastating outlook for affected patients and a certain helplessness in the general field of medicating brain related diseases. One current approach to address this apparent paradox of having an effective barrier shielding the brain from external harm and simultaneously providing accessibility of medication to specific targets is to apply focused ultrasound to the targeted brain region and thereby mechanically open the BBB [3]. Not fully understood however, is to 
which extent the surrounding healthy tissue is affected by that treatment [4]. Alternatively recent advances show that the permeability of the BBB can be reversibly modified through mild heating of only a few Kelvin [5] [6]. As inconsiderate microdrilling the skull of rodents was shown to cause heat damage in the underlying cortex [7], we hypothesize, that illuminating the brain with sufficient optical energy by $1470 \mathrm{~nm}$ infrared laser radiation will cause the same effect. At the same time, using light as heat source allows to apply simple optics to confine the effect and control it. As the penetration depth of 1470 $\mathrm{nm}$ in water is less than $0.9 \mathrm{~mm}$ [8], the superficial cortex of a rodent animal model was chosen to proof our hypothesis. To assess permeability changes of the BBB we used intravenously injected Evans Blue (EB) as it is a widely used marker for the BBB integrity. EB binds to serum albumin in the circulating blood which is prevented by an intact $\mathrm{BBB}$ from crossing into surrounding tissue.

\section{METHODS}

Prior to experiments, female Sprague-Dawley rats (290-310 g of weight) were initially anesthetized with Isofluran (5\%) and quickly mounted in a standard stereotactic frame on a heating pad. Isofluran narcosis was then reduced to $2.5-3.5 \%$. Reflexes were checked frequently. The head was shaved, the cranial skull bone was exposed by incision of the skin and cleaned with saline and hydrogen peroxide. The wound was held open and kept as dry as possible to avoid additional laser absorption by residual aqueous liquid. The surgical procedure was followed by venous tail vein access and injection of $4 \%$ Evans Blue (in $0.9 \% \mathrm{NaCl}, 2 \mathrm{ml} / \mathrm{kg}$ ). Successful injection was acknowledged by obvious blue coloration of the eyes and bare skin (ears, palms) within seconds. A resting period of 5 minutes assured thorough and uniform distribution of the dye in the rat's circulatory system.

For defined infrared laser illumination of various targets, we recently developed and presented a versatile experimental setup [9]. Briefly, the setup consists of a fiber-coupled infrared diode laser (1470 nm, 12 W, $200 \mu \mathrm{m}$ fiber, DILAS, Mainz, Germany) in combination with an optical lens assembly, which is mounted on a 3-axis micromanipulator for beam positioning. The setup provides a defined laser beam profile and therefore allows precise remote laser irradiation with minimum focus spot diameter $\mathrm{d}=538 \mu \mathrm{m}$ (1/e peak intensity threshold) and about $91 \mathrm{~mm}$ of operating distance, with about $10 \mathrm{~mm}$ depth of focus. For laser illumination of various locations on the exposed skull, the optical lens assembly was positioned vertically above the stereotaxic frame holding the anesthetized rat and the beam was focused perpendicularly on the partic- ular target location. In preliminary studies, five to six skull locations were illuminated with different laser output power, while the exposure time was held constant at 30 seconds. The laser was operated in continuous pulsed mode $(50 \mathrm{~Hz})$ and the optical output power was controlled by the pulse width (duty-cycle). During laser illumination, the local bone surface temperature was monitored by means of a high speed thermal camera (FLIR, A8303sc, custom macro lens, $165 \mathrm{fps}$ ). For temperature analysis, maximum temperature $T_{\max }$ was extracted for each frame. A schematic representation of the experimental setup is shown in Fig. 1. The laser illumination procedure was completed within 5-7 minutes, after which the rat was maintained under anesthesia for one hour to allow sufficient time for Evans Blue diffusion prior to intracardiac perfusion with $4 \%$ paraformaldehyde (PFA) and brain extraction.

\section{RESULTS}

\subsection{Localized heating by infrared laser illumination}

Infrared laser illumination of the exposed rat skull leads to localized bone surface heating with confined temperature profile. Fig. 2a shows a thermal image after 5 seconds of laser illumination at the right hemisphere of the skull. It clearly depicts the heated region, while the surrounding bone structure remains at baseline temperature (approx. $35.5^{\circ} \mathrm{C}$ ). Analysis of the temperature profile across the irradiated spot (along white horizontal line) revealed a Gaussian-like temperature distribution profile for all illuminated locations. Corresponding to the laser position, the obtained temperature profiles showed a symmetric circular shape and exhibited peak temperature in the center of the laser irradiated spot, with significant spatial decay with increasing radial distance. In Fig. 2a, the diameter of the circular heated spot on the skull was determined to be approx. $\mathrm{d}=1.3 \mathrm{~mm}$ (FWHM) with maximum temperature $T_{\max }=66^{\circ} \mathrm{C}$ after 5 seconds. Consistently, the maximum temperature $T_{\max }$ increased with exposure time. The observed skull surface temperature increased rapidly at the beginning (0-10 s) of laser illumination and then converged to a relatively stable level after 10-20 seconds. For the five skull locations targeted here, maximum temperature time courses during laser illumination are shown in Fig. 2c, that scaled with laser output power. With ongoing laser exposure time, the generated heat dissipated to the adjacent anatomical structures. Due to this propagation of heat to the surrounding tissue (bone, cortex), we observed the spatial temperature profile to widen with illumination time. Fig. $2 b$ illustrates the skull surface heating of the depicted exposure in Fig. 2 a after 25 seconds. In 

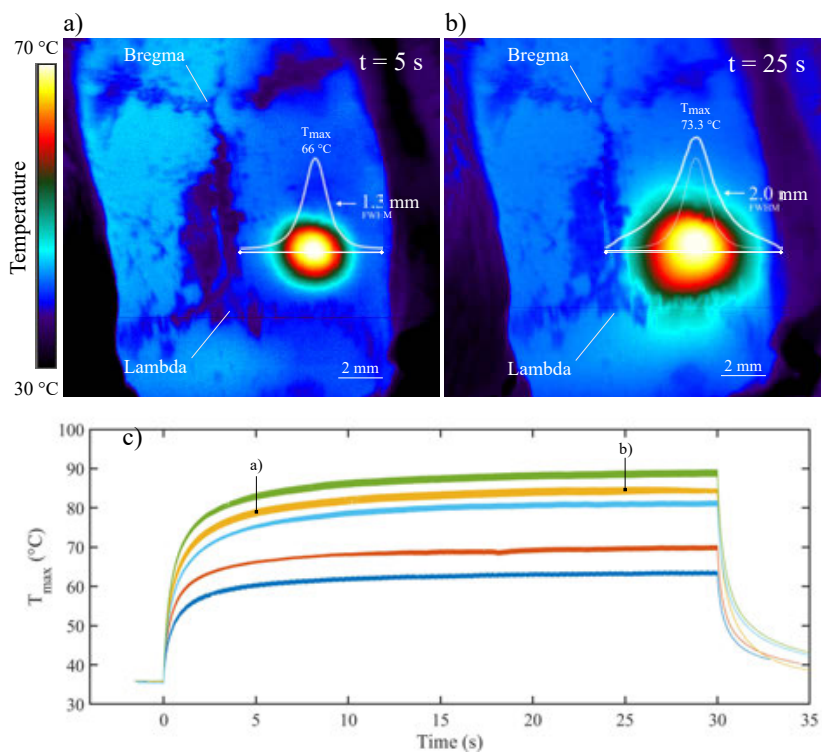

Fig. 2: Exemplary thermal camera images of an illuminated spot on the skull of a rat after a) $5 \mathrm{~s}$ and b) $25 \mathrm{~s}$ exposure time. Scale bar is $2 \mathrm{~mm}$. The thermal images correlate to the yellow temperature graph in c). From the spatial temperature profiles across the heated spot (white horizontal line), FWHM diameter was determined to be approx. $1.3 \mathrm{~mm}$ after $5 \mathrm{~s}$ (a), that enlarged to $2.0 \mathrm{~mm}$ after $25 \mathrm{~s}$ (b), compared to the $538 \mu \mathrm{m}$ laser spot size. After $25 \mathrm{~s}$, spatial temperature profile expansion was apparent. c) Temperature time course of five of the the illuminated spots on the skull.

addition to increased maximum temperature of $73.3{ }^{\circ} \mathrm{C}$ at this time, we observed a broadened, extensive spatial temperature distribution in the thermal image. The diameter of the heated spot after $25 \mathrm{~s}$ was determined to be approx. $\mathrm{d}=2.0 \mathrm{~mm}$. Consequently, our approach allows the controlled local heating of specific regions of diameter $\leq 2 \mathrm{~mm}$ on the skull for the investigation of localized Blood-Brain-Barrier opening under laser illumination.

\subsection{Localized BBB opening due to skull heating}

In the preliminary study presented here, we applied a wide range of laser induced heating parameters with final temperatures reaching up to $60-90{ }^{\circ} \mathrm{C}$ (see Fig. 2c). Laser heating by infrared illumination of the skull was applied to five distinct locations. Fig. 3 shows the fixated brain immediately after animal perfusion at the end of the experiment. The respective locations of laser illumination on the skull were determined on the cortex and marked with colored circles according to the temperature curves of the skull surface given in Fig. 2c. We observed pronounced blue spots, here on the right hemisphere, indicating Evans Blue diffusion into cortical tissue. We

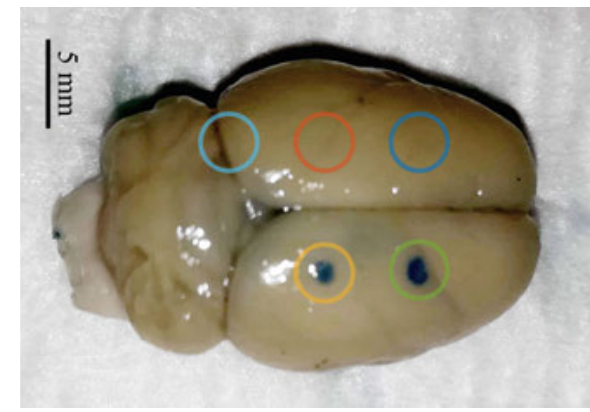

Fig. 3: Macroscopic view of the fixated and extracted rat brain. Projected locations below skull illumination points are marked in the same color as the temperature profiles in Fig. 2b. Note: Only for the highest achieved skull temperatures Evans Blue was found in the brain, depicting a BBB breakdown. Scalebar is $5 \mathrm{~mm}$.

interpret this observation to result from heat diffusion from the skull surface into the underlying cortical region and therefore localized opening of the Blood-Brain-Barrier, resulting in visible EB-loaded albumin penetration. The corresponding skull temperature for these two observed positive locations (green, yellow) was surprisingly high. According to Fig. 2c, only heating above $80^{\circ} \mathrm{C}$ showed a visible effect in the cortex. Despite achieving a skull temperature as similar as the positive spots, the light blue marked target location did not indicate the BBBbreach. We contribute this to the the different anatomical structure at the caudal bound of the cortex, causing a void to the inside of the skull leading to insufficient 'contact' and decreased heat transfer. This may have resulted in a lack of visual EB marking. However, without illuminating and monitoring the cortex directly (by means of a craniotomy), the actual cortical temperature needed for localized BBB-opening can only be guessed. From the fixated brain in Fig. 3, we observed a visible difference in size of the two EB spots. We estimated the diameter using image processing software, resulting in about 0.95 $\mathrm{mm}$ (yellow) and $1.1 \mathrm{~mm}$ (green). Qualitatively, the difference in diameter is in agreement with the temperature curves in Fig. $2 c$, since, despite equal exposure time, the relative temperature 'load' of the yellow location on the skull was lower compared to the green location. In histological brain slice analysis, unfortunately, the EB dye did unexpectedly wash out during the staining procedure, hence limiting detailed quantification of EB penetration. We will elaborate on this issue and consider the use of different marking agents in the future.

\section{CONCLUSION and OUTLOOK}

Our preliminary findings presented here corroborate the assumption that by localized infrared laser illumination of the skull surface, mediating heat diffusion to the particular un- 
derlying cortical region, local Brain-Blood-Barrier breakdown can be achieved. The observed EB penetration indicates local BBB opening in two locations that received high temperature load above $80{ }^{\circ} \mathrm{C}$, while other regions with less heating did not show visible EB colorization. Moreover, with the observed unequal Evans Blue spot diameters, it is safe to assume a correlation of skull heating (or emerging cortex temperature respectively) and the resulting extent of BBB opening. The 'successful' target temperatures were surprisingly high with the applied exposure time of 30 seconds being relatively short. Despite accurate temperature measurement of the skull surface temperature, the effective, 'secondary' cortical heating remains unclear. Therefore, in the future, we will perform craniotomy and direct laser illumination of the cortex. Further, we will investigate different modalities of e.g. long exposure times in combination with moderate heating temperatures and the resulting effects on BBB modification. In order to draw meaningful conclusions, experiments studying the BBB rely on suitable markers. EB has the advantage of being immediately visible offering direct feedback during an experiment making it an appropriate choice for the described proof of concept study. However, recently the selectivity of the dye towards plasma albumin has been questioned [10] which renders precise quantitative measurement impossible. Unbound EB molecules can, because of the smaller size, diffuse across the BBB of intact capillaries potentially leading to false positive results [11]. Therefore, in the course of extending the premise of the presented hypothesis we are planning to use Fluorescein isothiocyanate (FITC)-Dextran. Because of the high sensitivity of the fluorescent signal and low affinity towards plasma albumin it promisises to be a more suitable marker for the BBB integrity [12]. Additionally, FITC-dextran is available in different molecular weights and thereby more detailed assumptions can be made with regard to the physiology of the BBB [12] In further studies we are planning to characterize any damage caused by IR illumination with different parameters such as energy-duration of the stimulation. RNA sequencing is another method that can be utilized for damage assessment even under acute conditions. MRI scanning with various contrast agents could be of help in evaluating the illumination's physiological changes. An essential question that needs to be addressed in future works is how reliable and reversible the BBB can be opened and how long does it truly stay open to permit medication passage. The answer is of substantial importance especially when considering the important task the BBB performs in the brain.

Acknowledgment: The authors are indebted to Christian Münkel for expert technical assistance.

\section{Author Statement}

This work was supported by the German Federal Ministry of Research project FMT-13GW0230A and NEUROPHOS 13GW0155C. Authors state no conflict of interest. All animal experiments were performed with approval from the locally responsible Animal Welfare Committee with the Regierungspräsidium Freiburg in accordance with the guidelines of the European Union Directive 2010/63/UE (permit G17/80).

\section{References}

[1] Scott T. Brady and Leon Tai. Chapter 1 - cell biology of the nervous system. In Scott T. Brady, George J. Siegel, R. Wayne Albers, and Donald L. Price, editors, Basic Neurochemistry (Eighth Edition), pages 3 - 25. Academic Press, New York, 8th edition, 2012.

[2] Jann N Sarkaria et al. Is the blood-brain barrier really disrupted in all glioblastomas? a critical assessment of existing clinical data. Neuro-oncology, 20(2):184-191, 2017.

[3] Nir Lipsman et al. Blood-brain barrier opening in alzheimer's disease using mr-guided focused ultrasound. Nature Communications, 9(1):2336, 2018.

[4] Michael L Schwartz et al. Skull bone marrow injury caused by mr-guided focused ultrasound for cerebral functional procedures. Journal of neurosurgery, 1(aop):1-5, 2018.

[5] Eugene A Kiyatkin and Hari Shanker Sharma. Permeability of the blood-brain barrier depends on brain temperature. Neuroscience, 161:926-939, 2009.

[6] Seyed Nasrollah Tabatabaei et al. Remote control of the permeability of the blood-brain barrier by magnetic heating of nanoparticles: A proof of concept for brain drug delivery. Journal of Controlled Release, 206:49 - 57, 2015.

[7] Andrew J Shoffstall et al. Potential for thermal damage to the blood-brain barrier during craniotomy: implications for intracortical recording microelectrodes. Journal of neural engineering, 15(3):034001, 2018.

[8] Joseph A Curcio and Charles $C$ Petty. The near infrared absorption spectrum of liquid water. JOSA, 41(5):302-304, 1951.

[9] Paul Schlett et al. Experimental setup for the systematic investigation of infrared neural stimulation (ins). In World Congress on Medical Physics and Biomedical Engineering 2018, pages 77-81. Springer, 2019.

[10] Norman R. Saunders et al. Markers for blood-brain barrier integrity: how appropriate is evans blue in the twenty-first century and what are the alternatives? Frontiers in Neuroscience, 9:385, 2015.

[11] Reka Natarajan, Nicole Northrop, and Bryan Yamamoto. Fluorescein isothiocyanate (fitc)-dextran extravasation as a measure of blood-brain barrier permeability. Current Protocols in Neuroscience, 79(1):9.58.1-9.58.15.

[12] Angelika Hoffmann et al. High and low molecular weight fluorescein isothiocyanate (fitc)-dextrans to assess blood-brain barrier disruption: Technical considerations. Translational Stroke Research, 2(1):106-111, Mar 2011. 